\title{
Design considerations for an active soft orthotic system for shoulder rehabilitation
}

\section{Citation}

Kesner, S. B., L. Jentoft, F. L. Hammond, R. D. Howe, and M. Popovic. 2011. “Design Considerations for an Active Soft Orthotic System for Shoulder Rehabilitation." Annual International Conference of the IEEE Engineering in Medicine and Biology Society, Boston, MA, Aug. 30-Sept. 3, 2011: 8130-8134.

\section{Published Version}

doi:10.1109/IEMBS.2011.6092006

\section{Permanent link}

http://nrs.harvard.edu/urn-3:HUL.InstRepos:30782215

\section{Terms of Use}

This article was downloaded from Harvard University's DASH repository, and is made available under the terms and conditions applicable to Other Posted Material, as set forth at http:// nrs.harvard.edu/urn-3:HUL.InstRepos:dash.current.terms-of-use\#LAA

\section{Share Your Story}

The Harvard community has made this article openly available.

Please share how this access benefits you. Submit a story.

\section{Accessibility}




\title{
Design Considerations for an Active Soft Orthotic System for Shoulder Rehabilitation
}

\author{
Samuel B. Kesner, Student Member, IEEE, Leif Jentoft, Student Member, IEEE, \\ Frank L. Hammond III, Member, IEEE, Robert D. Howe, Senior Member, IEEE, and Marko Popovic
}

\begin{abstract}
Strokes affect over 750,000 people annually in the United States. This significant and disabling condition can result in paralysis that must be treated by regular sessions with a dedicated physical therapist in order to regain motor function. However, the use of therapists is expensive, in high demand, and requires patient travel to a rehabilitation clinic. We propose an inexpensive and wearable upper body orthotics system that can be used at home to provide the same level of rehabilitation as the current physical therapy standard of care. The system is composed of a soft orthotic device with an integrated cable actuation system that is worn over the upper body, a limb position sensing system, and an actuator package. This paper presents initial design considerations and the evaluation of a proof of concept system for shoulder joint rehabilitation. Through simulations and experimental evaluation, the system is shown to be adjustable, easily wearable, and adaptable to misalignment and anatomical variations. Insights provided by these initial studies will inform the development of a complete upper body orthotic system.
\end{abstract}

\section{INTRODUCTION}

$\mathrm{S}$ TROKE is the leading cause of long-term disability in the United States, affecting over 750,000 people annually [1]. Rehabilitation is the most effective method for restoring limb motor control after a serious stoke event [2],[3]. Standard rehabilitation methods require the dedicated attention of trained physical therapists to move the patients through a series of motion exercises to spur the regeneration of their neurophysiology and muscle control. This process is time consuming and expensive, requiring regular work with therapists to make any significant improvement [4],[5]. It is the goal of this work to design an affordable and wearable device that uses actuators and sensors for rehabilitation at home without a dedicated therapist.

Most previous actuated systems for upper body rehabilitation use rigid exoskeletons or rigid link manipulators [6], [7]. Soft devices, on the other hand, take advantage of natural anatomical structures, including joints and bones, to provide the device structure and determine the kinematic degrees of freedom (DOF). Ueda et al. attached pneumatic muscles to a wearable device to anthropomorphically apply actuation forces to the human

Manuscript received April 15, 2011.

S. B. Kesner, L. Jentoft, F. L. Hammond, and R. D. Howe are with the Harvard School of Engineering and Applied Sciences, Cambridge, MA, 02138 USA.

R. D. Howe is also with the Harvard-MIT Division of Health Sciences \& Technology, Cambridge, MA, 02139 USA. email: howe@seas.harvard.edu

M. B. Popovic is with Worcester Polytechnic Institute, Worcester, MA, USA, email: mpopovic@wpi.edu arm [8]. However, pneumatics are an expensive and complicated actuation method for a device to be used in the home. Agrawal et al. created a system of six shouldermounted motors and cables to actuate the arm in multiple DOF [9],[10]. This system's kinematics have been optimized to achieve a useful range of motion, however it is not adjustable to anatomical variations or easily wearable.

It is our goal to create an upper limb rehabilitation device that patients can use at home without any assistance. The device is an actuated soft orthotic that will move the patient through a serious of motions that mimic the exercises of a physical therapist. The advantages of our system are that it (1) is lightweight and wearable, (2) can adapt to misalignment and anatomical variation through sensing and control, and (3) is able to actuate the arm through a range of motion sufficient for rehabilitation. This paper presents the initial design and analysis of this system, which focuses on shoulder abduction-adduction and adjustability to anatomical variation and misalignment.

\section{DESIGN}

\section{A. System Concept}

The orthotic device concept presented here automatically adapts to joint topology, senses body movements, and reacts accordingly by creating appropriate assistive distributed force. The soft, actuated orthotic is depicted in Fig. 1. While the current system focuses on the shoulder, this technology will be applicable to the entire upper and potentially the lower extremities. The brace is lightweight, quiet, low-cost, robust, and designed for home and other non-clinical use. Instead of a rigid joint structure, the brace distributes forces and torques along the arm with soft attachment mechanisms. The brace operates as a conformal pad that the patient wraps around the limb adjacent to the joint and consists of a jointspecific number of muscle-like, cable driven series elastic actuators (SEAs) and a sensor network integrated into a wearable system [11].

Bowden cable systems connect the soft orthotic on the user's arm with a motor unit that can be either worn distally (attached to a belt or carried in backpack) or placed on a table. The ratio of applied torques (up to $\sim 20 \mathrm{Nm}$ for shoulder) to mass (less than $300 \mathrm{~g}$ ) of the brace worn proximally to the joint is maximized for the user's comfort by reducing forces on the arm. Each joint's mechanical DOF is matched with a redundant pair of antagonistic brace 


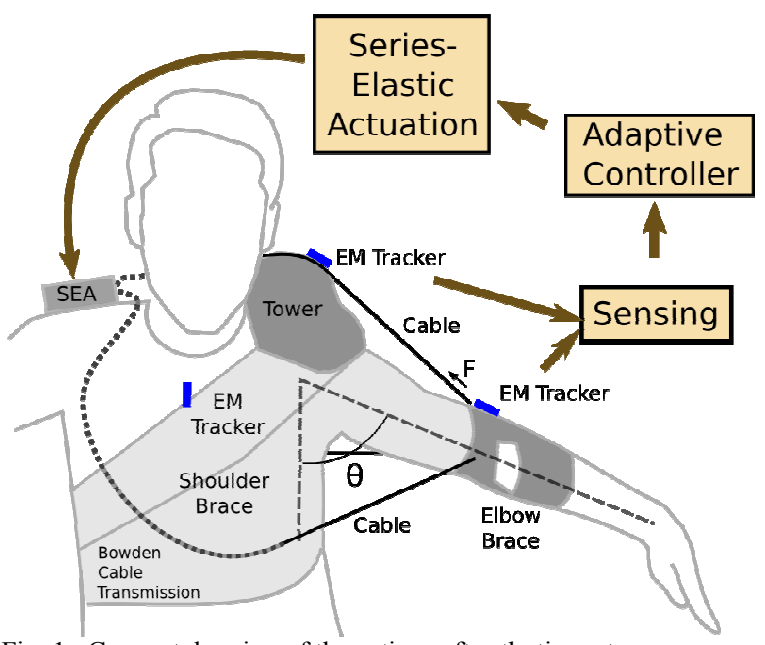

Fig. 1. Concept drawing of the active soft orthotic system

"muscles." Each antagonistic "muscle" pair provides pushpull functionality that minimizes the effects of transmission backlash. Additional "muscle" pairs will resolve the problem of joint (brace and human) misalignment and allow for the adaptability and fine joint torque and stiffness control.

Series elastic actuators (SEA) are a natural platform for force feedback control, as they add both biomimetic compliance and force sensing to the actuation system [11][13]. SEAs use elastic elements in series with actuators to provide compliance and force sensing through deformation measurements. These devices have proven successful in the context of lower limb exoskeletons and braces [14]-[16].

The first prototype of this soft orthotic device focuses on the shoulder joint. In this device, an actuated cable is attached to a brace on the upper arm, routed over an elevated "tower" on the shoulder, and connected to the actuation system (Fig. 1). The brace's adaptable virtual joint structure provides the same mechanical functionality as the human shoulder. The device concept, shown in Fig. 1, assists in shoulder abduction/adduction motion.

\section{B. System Simulation}

The design was first analyzed in simulation to better understand how the design parameters impact the system performance. A human arm model was constructed in order to calculate physical quantities such as moment due to gravity and to address cable attachment points within framework of single and double cable systems (Fig. 2).

The simplified three-segment arm model consists of the upper arm, forearm and hand. The arm segments' masses relative to body mass and the segments' center of mass locations relative to the arm length are modeled according to the average human [17],[18]. The arm model is then customized to best match the anatomical properties of the subject of the experimental study based on the subject's weight, arm length and the upper arm circumference proximal to the shoulder joint.

The model gravity moment, $\tau_{g}$, is obtained as function of the arm abduction/adduction angle and is the sum of the moments of individual segments. Each segment's lever arm

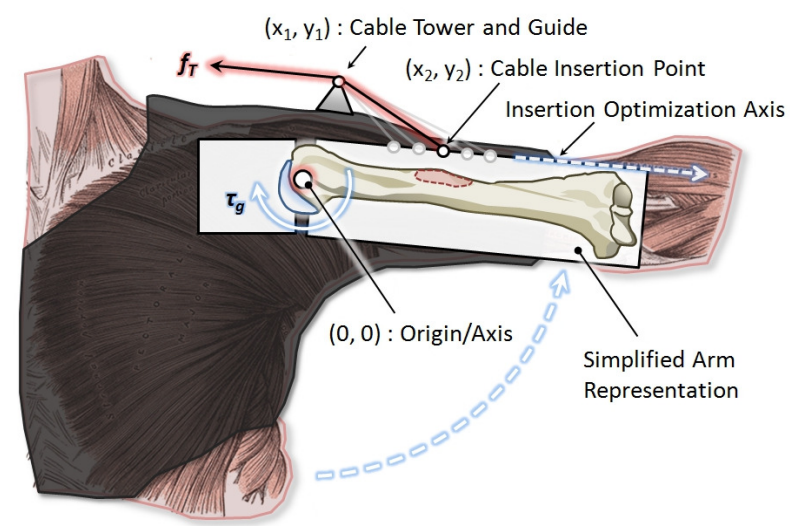

Fig. 2. Simulation model

is approximated by the horizontal distance between the arm's center of rotation and the segment's center of mass.

The coordinate frame origin is located at the glenohumeral joint and the coordinates of shoulder tower and the upper arm cable insertion point are $\left(x_{1}, y_{1}\right)$ and $\left(x_{2}, y_{2}\right)$, respectively (Fig. 2). Assuming a static balance condition, arm motion confined within the coronal plane and neglecting friction between the cable and guide, the cable tension is

$$
f_{T}=\frac{\sqrt{\left(x_{1}-x_{2}\right)^{2}+\left(y_{1}-y_{2}\right)^{2}}}{x_{2} y_{1}-y_{2} x_{1}} \tau_{g} .
$$

This model is used to validate experimental results in this study.

\section{ARM ABDUCtion Evaluation}

The first evaluation prototype of the proposed rehabilitation system focused on shoulder joint abductionadduction. This motion was selected because it requires one of the greatest forces from the orthotic system and thus represents the most critical actuation task. Fig. 3 presents a diagram of the initial shoulder orthotic prototype, which actuates the wearer's abduction-adduction DOF.

\section{A. Method}

The wearable components of the prototype device are composed of a shoulder stabilization brace, a foam and plastic tower on the shoulder, and a soft elbow brace that functions as a cable attachment point (Fig. 3). The device is actuated by cables connected from the area above the elbow, routed over the shoulder tower, and tensioned by another researcher to allow for safe adjustment of forces. The attachment and adjustment of the orthotic is controlled by Velcro straps on the arm and torso. The cable force is measured by a spring scale (Model T-20, Yamato Corp., Colorado Springs, CO, USA, Resolution: $1 \mathrm{~N}$ ) placed in series with the cable. The position and orientation of the user and orthotic device are measured in all six DOF by electromagnetic (EM) sensors (miniBIRD, Ascension Technology Corporation, Burlington, VT, resolution: $1 \mathrm{~mm}$, 1 degree). 

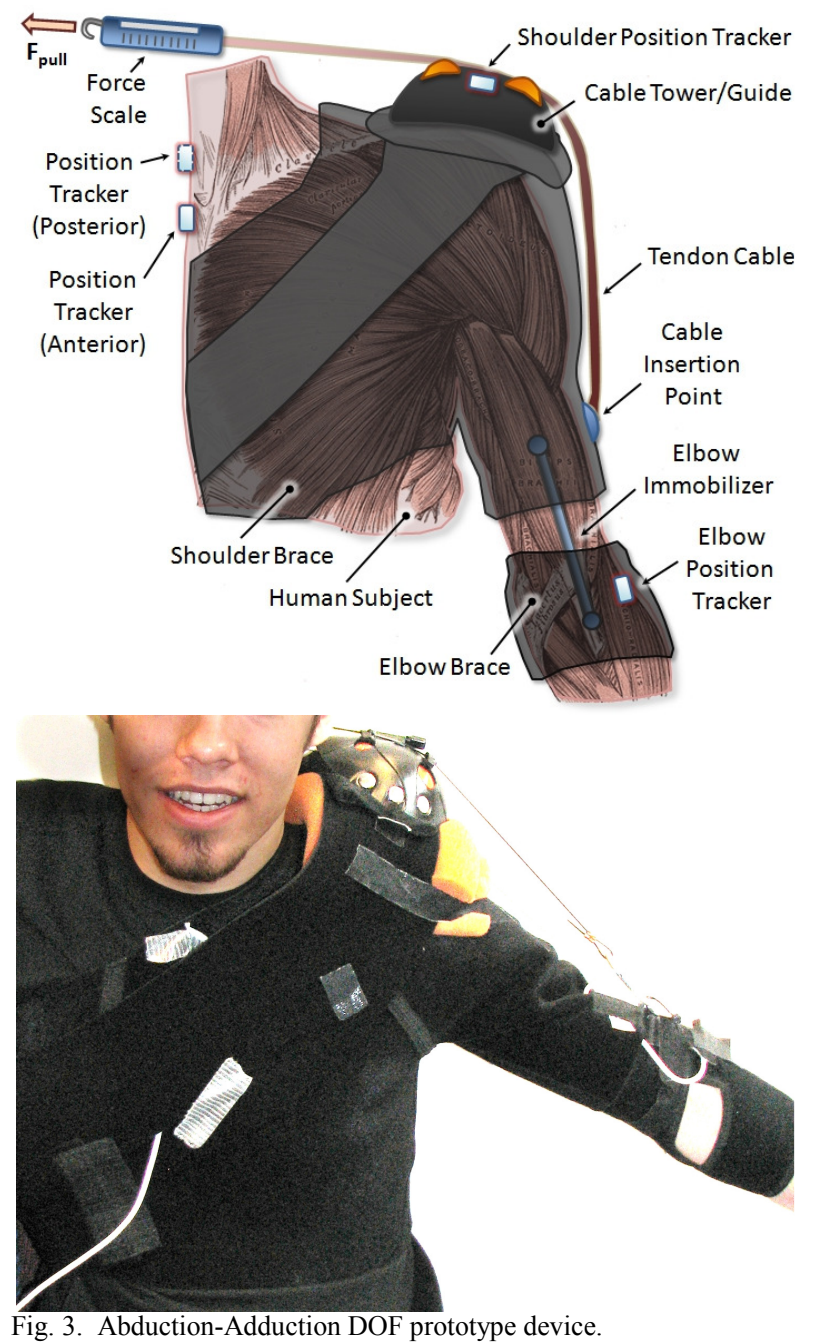

During the experiment, the test subject was braced in the sitting position while another researcher manually actuated the cable system (Fig. 3). This approach was taken to insure the safety of the subject during this initial evaluation phase of the project. As the arm was raised and lowered though a number of discrete elevation levels ( 20 levels for each raising-lowering cycle), the position of the subject's torso, shoulder tower, and forearm were recorded using the EM trackers. The cable tension was recorded using a precision spring scale for each position. This research was approved by the Harvard University IRB.

\section{B. Sensing}

Shoulder abduction angles were calculated using position data taken from the EM trackers (Fig. 4). An approximation of the humerus orientation vector $\overrightarrow{s e}$ was calculated as the difference between elbow tracker position $\left(x_{e}, y_{e}\right)$ and shoulder tracker position $\left(x_{s}, y_{s}\right)$. Angle reference vector $\overrightarrow{s t}$ was created by projecting the shoulder tracker position down in the y-direction by an arbitrary distance $d$ to a point $\left(x_{s}, y_{s}-d\right)$. The shoulder abduction angle $\theta_{a b d}$ was calculated in Eq. 2 as the difference between $\theta_{\text {meas }}$, the raw angle measured between the vector $\overrightarrow{s e}$ and angle reference vector $\overrightarrow{s t}$, and $\theta_{\text {ref }}$, the correction term for the angle

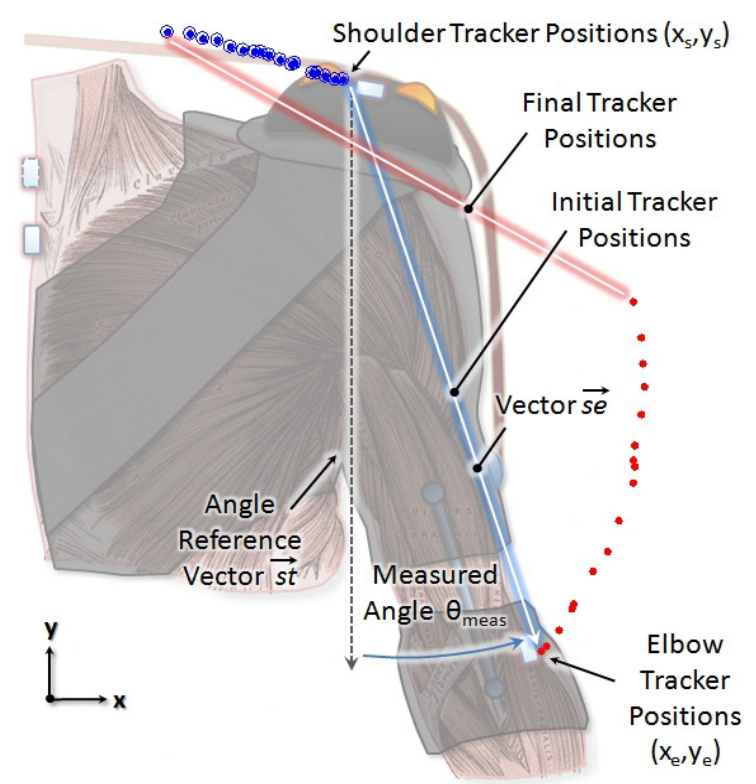

Fig. 4. Joint angle sensing using EM trackers

measured at the arm's rest position.

$$
\begin{aligned}
& \theta_{\mathrm{abd}}=\theta_{\text {meas }}-\theta_{\text {ref }} ; \theta_{\text {meas }}=\cos ^{-1}\left(\frac{\overrightarrow{s e} \cdot \overrightarrow{s t}}{|\overrightarrow{s e}||\overrightarrow{s t}|}\right) \\
& \text { C. Results }
\end{aligned}
$$

The shoulder abduction-adduction results are presented in Fig. 5. The device range of motion was constrained by the actuation and compliance limitations of the prototype. The results show some amount of force hysteresis in the movement due to friction. The contribution of overcoming static friction was experimentally found to be approximately 5-20 $\mathrm{N}$ of additional force on the system, depending on the angle of the arm and the amount of cable wrapped around the shoulder as predicted by the capstan effect. The friction effects seen in Fig. 5 fall within this range of friction values.

The experimental results also match well with the single cable model (Fig. 5). The cable tension was obtained from Eq. 1 with the shoulder cable guide and arm cable insertion points parameterized with their coordinates for the arm in the coronal plane orthogonal to the gravity vector (horizontal) and the coordinate frame origin coinciding with the arm's center of rotation:

$$
\begin{aligned}
& x_{1}=0.080 m, y_{1}=0.101 m, \\
& x_{2}=0.195 m, y_{2}=0.056 m
\end{aligned}
$$

The results in Fig. 5 fit the simulation results closely. The simulation predicts similar cable tensions to those found in the experiment, however without the hysteresis effects caused by the friction forces. The simulation matches the experimental results with an RMS error of 7.4 N.

\section{Misalignment Adjustment Evaluation}

To investigate device adaptability, a study was conducted to determine how to reduce unwanted twisting forces on the 


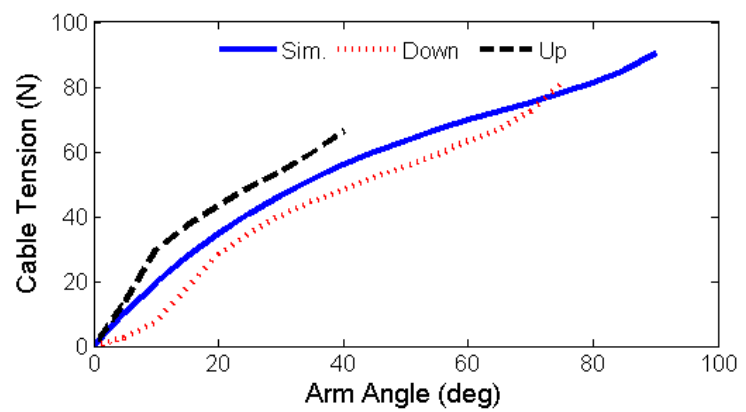

Fig. 5. Abduction-adduction experiment evaluation and model simulation results.

arm due to misalignment of the cable system caused by anatomical variation or user placement errors. A robot arm (Whole-Arm Manipulator (WAM), Barrett Technology, Cambridge, MA, USA) capable of direct joint torque measurement was used in place of human arm for this experiment.

\section{A. Method}

An orthotic brace was attached at the robotic elbow to support the robotic forearm, mass $1.6 \mathrm{~kg}$, in the vertical plane. The brace lifted the forearm against gravity and no active force and torque were generated by the robot at the elbow. The brace attachment orientation was varied about the axis of the upper arm through the angle range $\{-15 \mathrm{deg}$, $+15 \mathrm{deg}$ \} while the off-axis torques were measured by a $6-$ axis force-torque sensor (Gamma model, ATI Industrial Automation, Inc, Apex, NC, USA, $0.1 \mathrm{~N}$ resolution) mounted in series with the upper arm just above the elbow and below the brace.

\section{B. Results}

Two actuation methods were compared (Fig. 6). In the first method ("single actuation"), a single cable linked to the upper arm was tensioned until the arm reached the goal position of horizontal. In the second method ("double actuation"), two cables were attached to the upper arm brace, one on each side. Each cable was separately actuated so that the combined tension moved the arm to the desired position. Cable tensions were varied independently to minimize the undesirable off-axis joint torques. Double actuation achieved $\sim 50$ times better tolerance for the brace misalignments. See Fig. 6 for a comparison of empirical data for the two actuation strategy experiments.

\section{CABle InSERTION DESIGN CONSIDERATIONS}

The cable insertion point on the arm, as shown in Fig. 2, can be selected using a number of criteria. Two design criteria considered here are minimizing the overall cable tension and minimizing the forces applied into the shoulder joint that do not generate any torque.

1) Minimum Cable Tension

The cable insertion point that results in the minimal cable tension condition can be found for the $90^{\circ}$ angle configuration at the shoulder attachment, i.e. $\Varangle\left((0,0),\left(x_{1}, y_{1}\right),\left(x_{2}, y_{2}\right)\right)=\pi / 2$ in Fig. 2. The point is
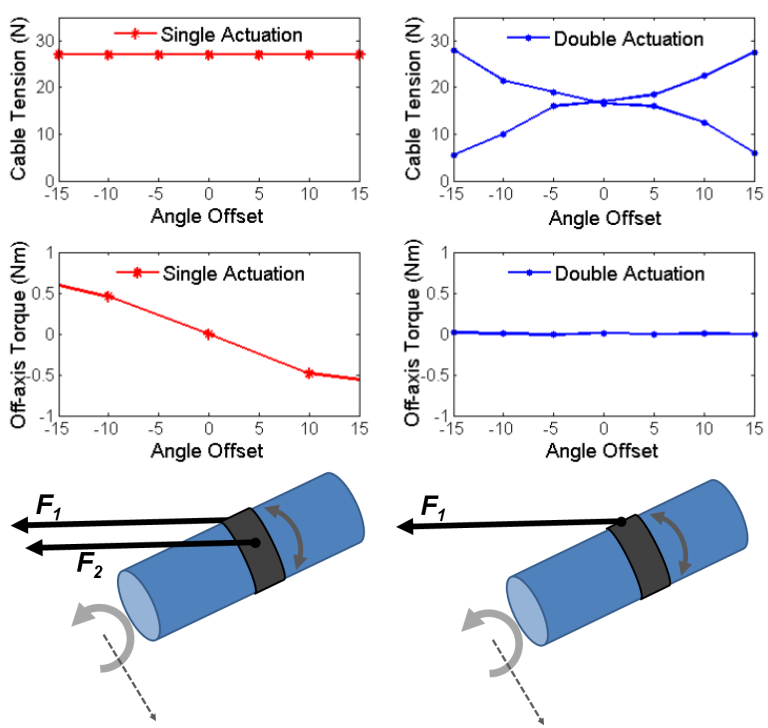

Fig. 6. Top: The cable tensions and arm torques generated by both the single and double cable actuation schemes. Bottom: The tow action methods examined in this study.

found by solving when the partial derivative of the cable tension, Eq. 1, with respect to $x_{2}$ is zero:

$$
x_{2}=x_{1}+\frac{y_{1}}{x_{1}}\left(y_{1}-y_{2}\right) \text {. }
$$

Thus for $\alpha_{a b d}=90^{\circ}, x_{1}=0.080 \mathrm{~m}, y_{1}=0.101 \mathrm{~m}$, and $y_{2}=0.056 \mathrm{~m}$ based on the experimental setup with the human subject in section III, the distance between the upper arm cable insertion point and the greater tubercle (i.e. roughly beginning of the humerus, the upper arm bone) is $x_{2}=0.136 \mathrm{~m}$ resulting in $\sim 6 \mathrm{~N}$ smaller maximal tension, within the considered arm angle range than the original cable insertion at $x_{2}=0.195 \mathrm{~m}$.

2) Minimum Force on Joint

The cable insertion point that minimizes the force applied into the shoulder joint (i.e. forces that do not apply a moment about the shoulder joint) can be found for the $90^{\circ}$ angle arm configuration using the following equation:

$$
x_{2}=\frac{x_{1}+\sqrt{x_{1}^{2}+4 y_{2}\left(y_{1}-y_{2}\right)}}{2} .
$$

This corresponds to $\Varangle\left((0,0),\left(x_{2 \perp}, y_{2 \perp}\right),\left(x_{1 \perp}, y_{1 \perp}\right)\right)=\pi / 2$ in Fig. 2. By substituting in the values used above for $x_{1}, y_{1}$, and $y_{2}$ for the arm angle $\alpha_{a b d}=90^{\circ}$, the distance between the upper arm cable insertion and greater tubercle is found to equal $x_{2}=0.104 \mathrm{~m}$. This insertion point removes unwanted forces that pull the arm toward trunk without generating any moments.

3) Comparison with Human Anatomy

The average deltoid muscle insertion into the proximal humerus in a study of 30 cadaver shoulders [19] shows reasonable agreement with above results. The mean distance from the greater tubercle to the proximal and distal insertion of the deltoid muscle in humans is $x_{\text {proximal }}=0.061 \mathrm{~m}$ and $x_{\text {distal }}=0.158 \mathrm{~m}$, respectively [19]. 


\section{DISCUSSION AND CONCLUSION}

The results above demonstrate the feasibility of the active soft orthotic system proposed in this paper. The abductionadduction experiments and simulation demonstrate that under $100 \mathrm{~N}$ of force is required to lift the arm up to a horizontal position. This can be achieved with a small motor $(<10 \mathrm{~W})$ and gear package for slower motions. Raising the arm represents one of the most significant actuation requirement on the system, thus the motors required for many of the other DOF can be even smaller and lighter than the one required for arm abduction. This result further supports the wearability and low-cost feature of the system.

The misalignment adjustment evaluation results support the need for adaptability of the orthotic system. If only a single cable is used, then any anatomical variation or alignment error will cause unintended forces and torques on the user's joints, resulting in discomfort. Through the use of intelligent SEA cable force and limb position sensing, the system will automatically adjust the cable tensions to compensate for any misalignments to reduce the unwanted forces on the user while still achieving the required level of rehabilitation. Redundant cables can be used in parallel, as in section IV, to compensate to torques on the joint, and also in series to adjust the virtual cable insertion point. By adding redundant collinear cables in the coronal plane with different insertion points, the system can adjust the virtual insertion point using the control system to actively minimize the various forces on the system discussed in the design considerations in section $\mathrm{V}$ and adjust for a range of arm sizes and anatomical variations.

The next steps for this project are to design and evaluate a complete soft shoulder orthotic that actuates the upper arm in all 3 DOF (abduction, rotation, and flexion). This system will be integrated with an inexpensive visual tracking system, such as the commercially available Microsoft Kinect $(<\$ 150)$, to investigate the possibility of optically tracking the device in 3D. In addition, we have conducted other preliminary work to demonstrate that a low cost flex sensor network can be integrated into an orthotic to provide joint angle and position information [20]. Advance control systems will also be explored to address reflexive disturbances and potentially larger forces associated with hypertonia due to spasticity after stroke. Finally, additional human studies will be conducted with a physical therapist to ensure the system can provided the necessary therapy for stroke rehabilitation.

\section{ACKNOWLEDGMENT}

The authors would like Dr. Krystyna Gielo-Perczak in the Department of Biomedical Engineering at Worcester Polytechnic Institute for the clinical insight and biomechanical background she provided on the topic of shoulder rehabilitation.

\section{REFERENCES}

[1] G. R. Williams, J. G. Jiang, et al, "Incidence and occurrence of total stroke", Stroke, vol. 30, 1999. pp.2523-2528.

[2] P Lum, D Reinkensmeyer, R Mahoney, "Robotic devices for movement therapy after stroke: current status and challenges to clinical acceptance," Topics in Stroke, 2002.

[3] C. D. Takahashi, "Robot-based hand motor therapy after stroke", Brain, vol. 131, 1997, pp 425-437.

[4] B. Kibler, "Shoulder rehabilitation: principles and practice", Clinical Supplement: The Shoulder, vol 30, 1998, pp. 40-50.

[5] R. Donatelli, "Physical Therapy of the Shoulder" Clinics in Physical Therapy, 2003.

[6] H. I. Krebs, N. Hogan, et al., "Overview of clinical trials with MITMANUS: a robot-aided neuro-rehabilitation facility”, Technology Health Care. vol. 7, 1999, pp. 419-23.

[7] L. E. Kahn, et al., "Robot-assisted reaching exercise promotes arm movement recovery in chronic hemiparetic stroke: a randomized controlled pilot study", J Neuroengineering Rehabil., vol. 3, 2006.

[8] J. Ueda, et al. "Individual Muscle Control Using an Exoskeleton Robot for Muscle Function Testing", IEEE Trans. On Neural Sys. And Rehab. Eng., vol, 18, n 4, 2010, pp 339-350.

[9] E. A. Brackbill, Y. Mao, S. K. Agrawal, et al., "Dynamics and Control of a 4-dof Wearable Cable-driven Upper Arm Exoskeleton", in proceedings of Int. Conf on Robotics and Automation", 2009.

[10] S. K. Agrawal, et al., "Optimization and Design of a Cable Driven Upper Arm Exoskeleton”, in proceedings of Int. Design Eng. Tech Conf. (IDETC/CIE), 2009.

[11] G. A. Pratt, M. M. Williamson, "Series elastic actuators," in Proc. of IEEE/RSJ Int. Conference on Intelligent Robots and Systems, 1995.

[12] G. A. Pratt, M. M. Williamson, P. Dilworth, J. Pratt J. and A. Wright, "Stiffness isn't everything," Experimental Robotics IV, Lecture Notes in Control and Information Sciences, 1997, vol. 223, 253-262, DOI: 10.1007/BFb0035216 1997

[13] J. Pratt, P. Dilworth, G. Pratt, "Virtual model control of a bipedal walking robot," in proc. of IEEE International Conference on Robotics and Automation, 1997

[14] H. Herr H., J. D. Paluska, P. Dilworth, "Artificial human limbs and joints employing actuators, springs, and variable-damper elements," Application number: 11/395,448 Publication number: US 2006/0249315 A1, Filing date: Mar 31, 2006. Publication date Nov 9 , 2006.

[15] H. Herr H., et al "Artificial ankle-foot system with spring, variable damping, and series-elastic actuator components," Application number: 11/495,140, Publication \#: US 2007/0043449 A1, U.S. Classification 623024000; 623052000 Filing date: Jul 29, 2006. Publication date Feb 22, 2007.

[16] J. F. Veneman, "Design and Evaluation of the LOPES Exoskeleton Robot for Interactive Gait Rehabilitation", IEEE Trans. On Neural Sys. And Rehab. Eng.., Vol. 15, 2007.

[17] A. R. Tilley A. R., H. Dreyfuss, The Measure of Man and Woman. Whitney Library of Design, Watson-Guptill Publications, New York, 1993.

[18] D. A. Winter, Biomechanics and Motor Control of Human Movement, Wiley, New York, 1990.

[19] S. J. Morgan, "The deltoid muscle: an anatomic description of the deltoid insertion to the proximal humerus", J. Orthop. Trauma, vol 20, pp. 19-21, 2006.

[20] A. Blumenau, D. O. Girardo, E. L. Lin, S. Mandala, and M. B Popovic, "Physics applied to post-stroke rehabilitation", AIP SPS award June 2011 interim report. (available at www.spsnational.org/ programs/awards/2011/ugr2011_Worcester.pdf) 